\title{
A furocoumarin, Imperatorin isolated from Urena lobata L. (Malvaceae)
}

\section{Keshab Ghosh}

Department of Chemical Technology, University College of Science \& Technology, Calcutta University, Kolkata - 700 009, India.

Fax 91-033-351-9755.

E-mail: keshab_g@yahoo.com

Received: 29 September 2003 / Accepted: 2 March 2004 / Published: 29 March 2004

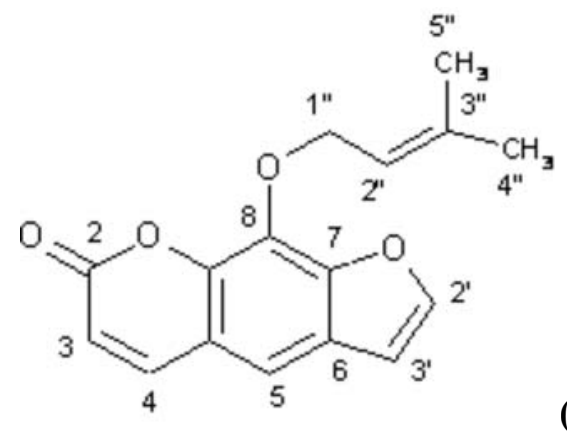

(I)

The plant, Urena lobata L. (Malvaceae) is reputed for its use in indigenous system of medicine. ${ }^{1}$ Phytochemical study of the aerial part of this plant showed the presence of mangiferin and quercetin. ${ }^{2}$ The roots have not been investigated so far for their active constituents. The isolation of imperatorin is being reported for the first time from this source.

Air-dried and pulverised roots $(1.5 \mathrm{~kg})$ of this plant were exhaustively extracted under reflux with benzene. The solvent was distilled off under the reduced pressure to yield $8 \mathrm{~g}$ of the extract which was then column chromatographed over silica gel. Elution of the column with petroleum-ether: benzene (4:3) eluate yielded an impure solid which on rechromatography over silica-gel column afforded a pure solid crystallized from petroleum-ether as crystals (I), yield: 34mg (0.0023 \%).

M.p. $98-99^{\circ} \mathrm{C}($ petroleum-ether); Optically inactive.

UV (1 $\left.1_{\max }, \mathrm{MeOH}, \mathrm{nm}\right): 301$ (log e 3.86), 266 (log e 3.97).

IR ( $\mathrm{n}_{\max }, \mathrm{KBr}, \mathrm{cm}^{-1}$ ): $3080(\mathrm{C}=\mathrm{C}), 1720$ (C=O of lactone ring), 1680, 1640, 1580 (aromatic),1440, 1220 (C-O-C stretching, asymmetric), 1150, 1085, 1030 (benzofuran), 990, 910 and 880.

${ }^{1}$ H-NMR (300 MHz, CDCl3): d: 7.69 (1H, d, J=2 Hz, H-2ф), 6.81(1H, d, J=2 Hz, H-3ф), 6.37(1H, d, J=9.6 Hz, H-3), 7.76 (1H, d, J=9.6 Hz, H-4), 7.36 (1H, s, H-5), 5.00 (2H, d, J=7 Hz, H-1²), 5.61 (1H, t, J= 7 Hz, $\left.\mathrm{H}-2^{2}\right), 1.72\left(3 \mathrm{H}, \mathrm{s}, \mathrm{Me}-4^{2}\right.$ or $\left.5^{2}\right), 1.74\left(3 \mathrm{H}, \mathrm{s}, \mathrm{Me}-4^{2}\right.$ or $\left.5^{2}\right)$. 
${ }^{13} \mathrm{C}-\mathrm{NMR}$ (75 MHz, CDCl3): 146.5 (C-2ф), 106.6 (C-3ф), 160.2 (C-2), 114.6 (C-3), 144.1 (C-4), 116.5 (C-4a), 113.0 (C-5), 125.6 (C-6), 148.1 (C-7), 125.6 (C-8), 139.2 (C-8a), 70.1 (C-1²), 119.8 (C-2²), 131.2 $\left(\mathrm{C}-3^{2}\right), 18.0\left(\mathrm{C}-4^{2}\right), 25.6\left(\mathrm{C}-5^{2}\right)$.

EIMS: m/z : $270[\mathrm{M}]^{+}\left(\mathrm{C}_{16} \mathrm{H}_{14} \mathrm{O}_{4}\right), 202$ (100\%), 174, 146, 145, 136, 117, 90, 89.

The ${ }^{1} \mathrm{H}-\&{ }^{13} \mathrm{C}$-NMR analyses were in consonance with the molecular architecture of imperatorin. ${ }^{3,4}$

\section{Acknowledgements}

Thanks are due to C.S.I.R., New Delhi, India for providing Senior Research Fellowship to K. Ghosh and to Centre of Advanced Study (CAS) on Natural Products, Department of Chemistry, Calcutta University for ${ }^{1} \mathrm{H}$ $\&{ }^{13}$ C-NMR spectra.

\section{References:}

1. "The Wealth of India : Raw Materials", Vol 10, Publications \& Information Directorate, C.S. I. R., New Delhi, 1976, p. 414-416.

2. Chem. Abstr. 1982, 96, 24699 t.

3. Bandhopadhyay, M.; Malik, S. B.; Seshadri, T. R. Indian J. Chem. 1971, 9, 731-732.

4. Levy, G. C. Topics in Carbon-13 NMR Spectroscopy 1976, 2, 110

(C) 2004 MDPI. All rights reserved. 